\title{
Effect of microbial inoculants and nitrogen fertilization on soil mites and collembola in cowpea crop, at Kafr el - Sheikh, Egypt.
}

\author{
Abou-Tayesh, M.A.M \\ Sakha Agricultural Research Station, Plant Protection Research Institute, ARC, Egypt
}

\begin{abstract}
Two field trials were conducted at Sakha Agric. Res. Station during the two successive summer growing seasons of 2010 and 2011. Cowpea plants (vigna unquiculata (L.) walp.) variety Dokki 331, were inoculated with micro symbiont. Bradyrhizobium sp along with urea fertilizer at 40 and $80 \mathrm{~kg} \mathrm{~N} / \mathrm{fed}$. applied in two equal doses : at 30and 45 days after sowing. The investigation lasted for three months in the two seasons of study aiming to survey the occurring soil predacious mites, miscellaneous mites and Collembola in four Nodulation and $\mathrm{N}-$ fertilization treatments : Nodulated $+50 \% \mathrm{~N}$, Nodulated $+100 \% \mathrm{~N}$, Non - Nodulated $+50 \% \mathrm{~N}$, and Non - Nodulated $+100 \% \mathrm{~N}$ under field conditions and the diversity between species under Nodulation and $\mathrm{N}$ - fertilization. Results revealed occurring of 8 species of predacious mite, belonging to 7 families in all Nodulators and $\mathrm{N}$ - fertilizers during the two seasons . while miscellaneous mite had 14 species belonging to 13 families in all Nodulators and $\mathrm{N}$ - fertilizers during the two seasons, except for Non - Nodulation $+100 \% \mathrm{~N}$ during seasons 2010, which had 13species belonging to 12 families. Also, 5 species of Collembola belonging to 5 families in both two seasons. Results indicated that the population density of the soil mite and Collembola increased with application of both microbial inoculants and N-fertilization, recording the highest figure at $80 \mathrm{~kg}$ $\mathrm{N} / \mathrm{fed}$. Amblyseius $s p$ and Cheyletus malaccensis (Oudmans) were the most dominant species under different applications of microbial inoculants and N-fertilization. While, Cheyletus eruditus (Oudmans )was the least species to be recorded.Pygmephorus sp. was the dominant species under cowpea field in the two seasons under different types of Nodulators and N-fertilizers. While, Galumna sp was the least one Proistoma sp. was the dominant species in the two seasons under different types of Nodulators and $\mathrm{N}$ - fertilizers , while, Onychiurus sp. Hypogastura sp. Tulbergi sp. and Entombyra sp. were least recorded species .

Diversity index values of predacious mite species were $(0.8456,0.8638,0.9330,0.8525)$ and $(0.8753$, $0.8874,0.8659,0.8866)$. While miscellaneous mite, were $(2.1411,1.0919,2.7336,1.0888)$ and $(1.1047,1.1169$, $1.1047,1.1096)$ on the other hand Collembola were $(0.6974,0.6966,0.6978,0.6985)$ and $(0.6977,0.6976$, $0.6981,0.6984)$ for Nodulated $+50 \% \mathrm{~N}$, Nodulated $+100 \% \mathrm{~N}$, Non - Nodulated $+50 \% \mathrm{~N}$, and Non - Nodulated $+100 \% \mathrm{~N}$ during seasons (2010 and 2011), respectively.
\end{abstract}

Keywords: microbial incoulants , nitrogen fertilization , mites , collembola , cowpea

\section{Introduction}

Cowpea is one of the most important leguminous vegetable crops in Egypt .Recently,its cultivated area was rapidly increased .However, the production of this crop has bean constrained by the limitation imposed by soil mites and Collembola. which cause serious damage. The total number of predacious mite, miscellaneous mite and Collembola species . differ among crops and proportion of species also varied (EL- Hawary et al.,1995). The relationship between species diversity and ecological processes can change quantitatively and qualitatively with environmental context (Cardinale $\&$ Nelson,1999) .Survey of predacious mite and miscellaneous mite and Collembola associated with cowpea plants were reported by( Hassan et al.,1985 and Abdel - Alim 1994) .it was stated that it can derive nitrogen from soil ,fertilizer and through dinitrogen - fixation by symbiosis with the specific micro partner Bradyrhizobium sp ,Increase of plant nitrogen content to certain level was found to induce the severity of infestation with major cowpea pests such as soil mites and Collembola (El-Kifl, 1957 and Abo-korah et al.,1985 ).

Investigation on soil mites is necessary according to their importance in improving soil fertility .Moreover, some soil mites act as predators and /or parasites for different stages of injurious species of mites (El-Kifl, 1957\& 1968, Tadros, 1975 and Boraei et al.,1994 ). Soil fauna always flourish and increase under different crops receiving nitrogen fertilization, while higher nitrogen level more than the recommended doses reduce their population density (Abo Korah et al.,1984-1985 a \& Tadros, 1975, Sharshir 1986 , Rosche 1992 and Gamieh \& Saadon 1995).

The objective of the present work aimed to study the effect of application of microbical inoculants of cowpea seeds with the micro symbiont Bradyrhizobium sp ., two rates of nitrogen fertilizer on soil mites and Collembola on cowpea plants and to see the species diversity as related to cowpea variety Dokki 331 in Egypt. 


\section{Materials and methods}

Two field trials were conducted at Sakha Agricultural Research Station, Kafr EL- Sheikh ,Egypt . in the two seasons of 2010 and 2011; Cowpea variety Dokki 331 was sown on June $1^{\text {st }}$ 2010 and in May $28^{\text {th }}, 2011$ in clay -loamy alluvial soil in a Split - Split Plot design with four replications. Each sub sub-plot $\left(21 \mathrm{~m}^{2}\right)$ contained 10 rows . The main -plots were randomly assigned by microbial inoculants application for seeds (or not )with the micro symbiont Bradyrhizobium sp.using a peat -based inoculum $\left(8.40 \times 10^{8} \mathrm{cfu} / \mathrm{g}\right)$ at $200 \mathrm{~g}$ inoculum $/ 40 \mathrm{~kg}$ seeds. The plots were irrigated Just after sowing.The sub -plots were assigned to the urea fertilization $(46 \% \mathrm{~N})$ at 40 and $80 \mathrm{~kg} \mathrm{~N} / \mathrm{fed}$ applied in two equal doses 30 and 45 days after sowing.

The used cultural practices (treatments)were as follows:

Nodulated $+50 \% \mathrm{~N}$ - fertilization $(40 \mathrm{~kg} /$ feddan $)$

Nodulated $+100 \% \mathrm{~N}$ - fertilization $(80 \mathrm{~kg} /$ feddan $)$

Non- Nodulated $+50 \% \mathrm{~N}-$ fertilization (40kg/feddan)

Non- Nodulated $+\quad 100 \% \quad$ Nfertilization $(80 \mathrm{~kg} /$ feddan )

Sampling started one week after sowing .An area of one Feddan was chosen for carrying out the present study.Samples were taken weekley by the use of a steel cylinder of $10 \mathrm{~cm}$ diameter and $20 \mathrm{~cm}$ depth as described by EL-kifl (1957) was used in the present investigation. They obtain about $1571.5 \mathrm{cc}$ of soil . Four samples were taken at random on every sampling date.

Every soil sample was put in a special plastic bag, tightly closed recording the needed data on each bag and carried directly to the laboratory for the extraction of organisms. Separation of organisms took place by the electric use of batteries of modified tullgren funnels adapted with 40 Watt bulbs for 48 hours. Organisms were collected in Petri - dishes containing water. Identification of organisms was done by help of a binocular microscope. At every sampling date, four replicates were taken and mixed together thoroughly. From those thoroughly mixed four samples, $0.75 \mathrm{~kg}$ was taken to represent that date and location of soil sample.

Shannon-Weaner diversity index (S.W.I.) was used to measure diversity of arthropod pest species as it is one of the most commonly used (Price, 1984). The Shannon-Weaner index was calculated according to the following equation:

\section{$\mathrm{Hs}=\sum_{\mathrm{i}}^{\mathrm{s}} \mathrm{Pi} \log \mathrm{Pi}$}

$\mathrm{Hs}=$ The symbol for the amount of diversity in a group of species in this case, the category of classification used in the species (hence the sub-scripts) but other categories could be used as well, it may be applicable to families, order, ... etc.

$\mathrm{S}=$ Number of species with sample,

$\mathrm{Pi}=$ The proportion of the $1^{\text {st }}$ species in the total sample, it measures the relative abundance and ranges between 0.00 to 1.00

$\log _{\mathrm{e}}=$ Natural logarithm. The negative sing is added to make the come out positive value $=2.718$.

The function was derived independently by Shannon and Weaner and is sometimes mislabeled as the Shannon-Weaner function in the ecological literature (Kerbs, 1978).

\section{Results and discussion}

\section{Survey studies:}

As shown in Table (1), the first category was the total recorded predacious mite species were 8 species from Nodulated $+50 \% \mathrm{~N}$ - fertilization, Nodulated $+100 \% \mathrm{~N}$ - fertilization, Non- Nodulated $+50 \% \mathrm{~N}$ - fertilization and Non- Nodulated $+100 \%$ $\mathrm{N}$ - fertilization during seasons 2010\& 2011 . While, were 14 species were recorded from miscellaneous mite belonging to 13 families in both Nodulated + $50 \% \mathrm{~N}-$ fertilization, Nodulated $+100 \% \mathrm{~N}-$ fertilization, and Non- Nodulated $+50 \% \mathrm{~N}-$ fertilization that 13 miscellaneous mite species belonging to 12 families during season 2010. On the other hand, during season 2011 , it could be stated that 14 miscellaneous mite species belonging to 13 families were recorded from Nodulated and $\mathrm{N}$ fertilization above mentioned. Finally the springtails (Collembola) were represented by 5 species in all Nodulation and N- fertilization during 2010\&2011. Population of these fauna in cultivated soil with a crop is affected by many factors ;i.e, cultivated crop, water content, soil porosity, Nodulation and $\mathrm{N}$ fertilization, organic matter, content, total soluble salts and mechanical analysis (Abou -Tayesh, 2008,Abou-Tayesh et al.2013and Gamieh et al .1998).

\section{Population density of predacious\& miscellaneous mite and collembolan species in soil on four Nodulation and $\mathbf{N}$ - fertilization in cowpea field:}

\section{1. predacious mites: A- Season 2010:}

In Table (1), the dominant recorded species was Amblyseius sp. reaching $(16.51 \%)$ of the total predacious mite in Nodulation $+50 \% \mathrm{~N}$ - fertilization while ,the least recorded one was Cheyletus eruditus (Schrank ) reaching (0.54\%), Macracheles sp . came in second rank, Rhodacarus sp $(15.15 \%)$.was the third in rank.All other species came in -between prep (those records it in a descending magnitude , the following picture would indicate that, Cheyletus malaccensis (oudmans) (14.88\%), Bdella sp. (14.47\%), Cunaxa capreolus (Berlese) $(14.21 \%)$ and Blattioscius sp $(7.85 \%)$. while, in Nodulation + 
$100 \% \mathrm{~N}$ - fertilization, the prevalent recorded species was Amblyseius sp. reaching (17.51\%) of the total predocious mite, while , the least one was Cheyletus eruditus (Oudmans )reaching (2.09\%) .Cunaxa caprealus (Berlese) came the second in rank, Bdella $s p(15.16 \%)$ the third in rank .All other species came in between preparing it in a descending magnitude .The following picture indicated that, Cheyletus malaccensis (oudmans) (13.41\%), Macrocheles $s p(12.72 \%), \quad$ Rhodacarus sp .(12.36\%) and Blattisocius sp (9.58\%) . While, in NonNodulation+ $50 \% \mathrm{~N}$ - fertilization, as shown , dominant recorded species was Amblyseius sp. reaching $(17.56 \%)$ of the total predocious mite, while ,the least one recorded was Cheyletus eruditus (Oudmans ) reaching $(0.75 \%)$. Bdella sp. came second in $\operatorname{rank}(17.55 \%)$ and Cheyletus malaccensis (Oudmans) (16.04\%), the third in rank .All other species came in-between preparing those records it in a descending magnitude, the following picture indicated that, Rhodacarus sp .(14.90\%), Macrocheles sp. (14.72\%) and Blattisocius sp $(6.98 \%)$. Finally, in Non- Nodulation+ $100 \%$ Nfertilization the dominant recorded species was Amblyseius sp. reaching (16.56\%) of the total predacious mites, while ,the least one was Cheyletus eruditus (oudmans ) reaching $(0.91 \%)$. Bdella sp.(16.11\%) came second in rank and Cheyletus malaccensis (Oudmans) (14.89\%), the third in rank .All other species came in-between, and may be arranged records it in a descending magnitude as follows, Rhodacarus sp .(14.58\%),Macrocheles sp.(14.28\%) and Blattisocius sp. (8.51\%).

\section{B- Season 2011:}

Data in Table (1) show that in Nodulation + $50 \% \mathbf{N}$ - fertilization, the dominant recorded species was Amblyseius sp. reaching (15.67\%) of the total predacious mite, while, the least one recorded was Cheyletus eruditus (Oudmans) reaching (3.49\%). Bdella sp and Macrocheles sp . came second in rank . Cheyletus malaccensis (Oudmons) (14.43\%) was the third in rank followed by Cunaxa capreolus (Berlese) and Rhodacarus sp (13.85\%) the fourth in rank , then , Blattiosocius sp. (8.67\%) . In Nodulation $+100 \% \mathrm{~N}$ - fertilization, the prevalent recorded species was Amblyseius sp reaching (16.03\%) of the total predocious mite, while, the least one was Cheyletus eruditus (Oudmans )reaching (5.80\%). Bdella sp came second in rank followed by , Cunaxa caprealus (Berlese) (14.72\%).All other species came in -between preparing it in a descending magnitude as follows, Cheyletus malaccensis (Oudmans) (13.72\%), Mocrocheles sp.(12.99\%), Rhodacarus sp .$(11.84 \%)$ and Blattisocius sp $(9.72 \%)$. While, in Non- Nodulation+ $50 \% \mathrm{~N}$ - fertilization, as shown , that dominant recorded species was Amblyseius $s p$ reaching $(16.12 \%)$ of the total predacious mite, while ,the least one recorded was Cheyletus eruditus (Oudmans )reaching $(2.41 \%)$, Bdella sp. came second in rank, Cheyletus malaccensis (oudmans) $(15.81 \%)$, the third in rank. All other species came in-between to be arrange in a descending magnitude, as; Macrocheles $\mathrm{sp}(15.18 \%)$ Rhodacarus $\mathrm{sp}$ .(13.93\%), Cunaxa caprealus (Berlese) (11.83\%) and Blattisocius sp (8.69\%) . While in NonNodulation+ $100 \% \mathrm{~N}$ - fertilization, the dominant record species was Cheyletus malaccensis (Oudmans) reaching $(15.21 \%)$ of the total predacious mite, while, the least one was Cheyletus eruditus (Oudmans)(5.38\%) .

\subsection{Miscellaneous mites:}

\section{A.season 2010}

Data presented in Table (1) revealed that the most dominant miscellaneous mite in Nodulation+ $50 \% \mathrm{~N}$ - fertilization, was pygamephorus sp. reaching $(13.59 \%)$ of the total miscellaneous mites, While, the least recorded one was Galumna sp. reaching $(0.03 \%)$, Tydeus sp. came second in rank (10.38\%), Tyrophagus putrescentiae (Schrank) $(9.27 \%)$ had the third rank . All other species came in-between. The obtained data indicated that Tarsonemus sp.(8.76\%), Kleemannia plumosus (Oudmans)( 8.27\%), , lohmannia sp.(8.17\%), Phthiracaeus sp.( 7.95\%), Scheloribates sp.(7.92\%), Epilohmannia sp.(7.66\%), Oribatula sp. (6.85\%), Haplozetes sp (4.70\%), Hypochthonus sp. (3.24\%) and Belba sp (3.17\%), while in Nodulation+ $100 \%$ $\mathrm{N}$ - fertilization, the most prevalent recorded species was Pygmephorus sp.(12.43\%), of the total miscellaneous mite, while, the least one was Galumna sp.(0.15\%). While in Non- Nodulation+ $50 \% \mathrm{~N}$ - fertilization, the prevalent record species was Pygmephorus sp. reaching (11.89\%), of the total miscellaneous mites, while, the least one was Galumna sp. with $(0.05 \%)$. while in NonNodulation $+100 \% \mathrm{~N}$ - fertilization, as indicated, the dominant recorded species was Pygmephorus $s p$. reaching $(12.56 \%)$, of the total miscellaneous mites, while, the least one was Belba sp. with (2.85\%).

\section{B. season 2011}

Data in Table (1) show that the most dominant species in Nodulation+ $50 \% \mathrm{~N}$ - fertilization, Nodulation+ $100 \% \quad \mathrm{~N}-$ fertilization, NonNodulation+ $50 \% \mathrm{~N}$ - fertilization, and Non Nodulation+ $100 \% \quad \mathrm{~N}-$ fertilization was pygmephorus sp. with $(11.30 \%, 9.96 \%, 10.14 \%$ and $10.35 \%$ ) respectively of the total miscellaneous mite, while, the least recorded was Galumna sp. reaching $(0.29 \%, 0.93 \%, 0.28 \%$ and $0.35 \%)$ respectively.

\subsection{Collembola:}

\section{A.season 2010}

Results represented in table (1) show that, in Nodulation+ $50 \% \mathrm{~N}$ - fertilization, the dominant recorded species was proistoma sp. with(23.17\%) of the total springtails, while the least one was 
Onychurius sp. with(19.07\%).In Nodulation+ 100\% $\mathrm{N}$ - fertilization, the most prevalent recorded species was proistoma sp. with $(23.76 \%)$ of the total springtails, while, the least one was Entombyra sp. reaching(18.12\%).In Non- Nodulation+ $50 \%$ Nfertilization, the dominant recorded species was proistoma sp. with $(21.83 \%)$ of the total spring tails, while the least one was Hypogastrura sp .reaching $(17.41 \%)$.In Non-Nodulation+ $100 \% \mathrm{~N}$ - fertilization, the most prevalent recorded species was proistoma sp. with $(21.12 \%)$ of the total springtails, while the least one was Entombyra sp. reaching(19.00\%).

\section{B. season 2011:}

Data in table (1) show that, the most dominant species in Nodulation+ $50 \% \mathrm{~N}$ - fertilization , was proistoma $s p$ with $(23.04 \%)$ of the total springtails, while the least one was Tulbergi $s p$ with(19.12\%).In Nodulation+ $100 \% \mathrm{~N}$ - fertilization, the most prevalent recorded species was proistoma $s p$ with $(22.83 \%)$ of the total spring tails, while the least one was Entombyra sp with (18.65\%).In NonNodulated $+50 \% \mathrm{~N}$ - fertilization, the most dominant species was proistoma $s p$ reaching $(21.65 \%)$ of the total spring tails, while the least one was Hypogastrura sp reaching (18.27\%) .In NonNodulation+ $100 \% \mathrm{~N}$ - fertilization, the most dominant species was proistoma $s p$ with $(21.71 \%)$ of the total spring tails, while the least one was Hypogastrura sp reaching (19.12\%) .

Table 1. Mean numbers and percentages of predacious mite, miscellaneous mite and collembolan species sampled from soil of cowpea variety Dokki 331 during 2010 and 2011 seasons at Kafr El-Sheikh as affected by nodulation and fertilization .

\begin{tabular}{|c|c|c|c|c|c|c|c|c|c|c|}
\hline \multirow{3}{*}{\multicolumn{2}{|c|}{ Family }} & \multirow{3}{*}{ Species } & \multicolumn{8}{|c|}{ Season 2010} \\
\hline & & & \multicolumn{2}{|c|}{$*$} & \multicolumn{2}{|c|}{$* *$} & \multicolumn{2}{|c|}{$* * *$} & \multicolumn{2}{|c|}{$* * * *$} \\
\hline & & & Mean & $\%$ & Mean & $\%$ & Mean & $\%$ & Mean & $\%$ \\
\hline \multirow{8}{*}{ 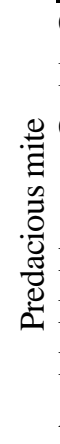 } & Cunaxidae & Cunaxa capreolus(Berlese) & 10.50 & 14.21 & 19.70 & 17.16 & 6.10 & 11.51 & 9.30 & 14.13 \\
\hline & Bdellidae & Bdella sp. & 10.70 & 14.47 & 17.40 & 15.16 & 9.30 & 17.55 & 10.60 & 16.11 \\
\hline & Cheyletidae & Cheyletus malaccensis (Oudmans & 11.00 & 14.88 & 15.40 & 13.41 & 8.50 & 16.04 & 9.80 & 14.89 \\
\hline & & Cheyletus eruditus(Oudmans & 0.40 & 0.54 & 2.40 & 2.09 & 0.40 & 0.75 & 0.60 & 0.91 \\
\hline & Phytoseiidae & Amblyseius sp. & 12.20 & 16.51 & 20.10 & 17.51 & 9.30 & 17.56 & 10.90 & 16.56 \\
\hline & Macrochelidae & Macrocheles sp. & 12.21 & 16.37 & 14.60 & 12.72 & 7.80 & 14.72 & 9.40 & 14.28 \\
\hline & Rhadacaridae & Rhodacarus sp. & 11.20 & 15.15 & 14.20 & 12.36 & 7.90 & 14.90 & 9.60 & 14.58 \\
\hline & Ascidae & Blattiosocius sp. & 5.80 & 7.85 & 11.00 & 9.58 & 3.70 & 6.98 & 5.60 & 8.51 \\
\hline \multirow{14}{*}{ 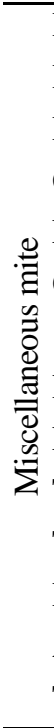 } & Haplozetidae & Haplozetes sp. & 14.50 & 4.70 & 28.70 & 6.13 & 10.30 & 5.08 & 15.80 & 5.62 \\
\hline & Hypochthoniidae & Hypochthonus sp. & 10.00 & 3.24 & 16.20 & 3.46 & 7.20 & 3.55 & 10.30 & 3.66 \\
\hline & Domacidae & Belba sp. & 9.80 & 3.17 & 13.50 & 2.88 & 3.90 & 1.92 & 8.00 & 2.85 \\
\hline & Galumnidae & Galumna sp. & 0.10 & 0.03 & 0.70 & 0.15 & 0.10 & 0.05 & 0 & 0 \\
\hline & Lohmannidae & Lohmannia sp. & 25.20 & 8.17 & 37.70 & 8.05 & 15.80 & 7.79 & 21.70 & 7.72 \\
\hline & Oribatulidae & Oribatula sp. & 21.10 & 6.85 & 31.80 & 6.79 & 14.70 & 7.25 & 20.50 & 7.29 \\
\hline & & Scheloribates sp. & 24.40 & 7.92 & 41.00 & 8.75 & 16.30 & 8.04 & 22.20 & 7.90 \\
\hline & Phthircaridae & Phthiracarus sp. & 24.50 & 7.95 & 43.70 & 9.33 & 15.90 & 7.85 & 21.90 & 7.79 \\
\hline & Epilohmanidae & Epilohmania sp. & 23.60 & 7.66 & 37.50 & 8.01 & 17.70 & 8.74 & 21.30 & 7.58 \\
\hline & Tarsonemidae & Tarsonemus sp. & 27.00 & 8.76 & 37.70 & 8.05 & 18.80 & 9.27 & 26.80 & 9.54 \\
\hline & Tydeidae & Tydeus sp. & 32.00 & 10.38 & 46.70 & 9.97 & 19.30 & 9.53 & 25.90 & 9.22 \\
\hline & Pygmephoridae & Pygmephorus sp. & 41.90 & 13.59 & 58.20 & 12.43 & 24.10 & 11.89 & 35.30 & 12.56 \\
\hline & Ameroseiidae & Kleemannia plumosus(Oud). & 25.50 & 8.27 & 31.40 & 6.70 & 18.20 & 8.98 & 23.20 & 8.25 \\
\hline & Tyroglyphidae & Tyrophagus putrescentiae (Sch). & 28.60 & 9.27 & 43.50 & 9.28 & 20.30 & 10.02 & 28.00 & 9.96 \\
\hline \multirow{5}{*}{$\begin{array}{l}\frac{\pi}{8} \\
\text { है } \\
\text { 0 } \\
0\end{array}$} & Onychiuridae & Onychiurus sp. & 54.50 & 19.07 & 62.80 & 18.29 & 32.10 & 20.25 & 52.00 & 20.45 \\
\hline & Sphaeridae & Tulbergia sp. & 54.80 & 19.18 & 68.80 & 20.04 & 32.20 & 20.31 & 50.50 & 19.86 \\
\hline & Hypogastruridae & Hypogastrura sp. & 55.10 & 19.28 & 67.90 & 19.77 & 27.60 & 17.41 & 49.70 & 19.55 \\
\hline & Poduridae & Proistoma sp. & 66.20 & 23.17 & 81.60 & 23.76 & 34.60 & 21.83 & 53.70 & 21.12 \\
\hline & Entombyridae & Entombyra sp. & 55.10 & 19.28 & 62.20 & 18.12 & 32.00 & 20.18 & 48.30 & 19.00 \\
\hline
\end{tabular}


Table 1. Continue

\begin{tabular}{|c|c|c|c|c|c|c|c|c|c|c|}
\hline & \multirow[t]{3}{*}{ Family } & \multirow[t]{3}{*}{ Species } & \multicolumn{8}{|c|}{ Season 2011} \\
\hline & & & \multicolumn{2}{|c|}{$*$} & \multicolumn{2}{|c|}{$* *$} & \multicolumn{2}{|c|}{$* * *$} & \multicolumn{2}{|c|}{$* * * *$} \\
\hline & & & Mean & $\%$ & Mean & $\%$ & Mean & $\%$ & Mean & $\%$ \\
\hline \multirow{8}{*}{ 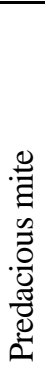 } & Cunaxidae & Cunaxa caprelous(Berlese) & 19.00 & 13.85 & 38.30 & 14.72 & 11.30 & 11.83 & 21.50 & 13.62 \\
\hline & Bdellidae & Bdella sp. & 20.60 & 15.01 & 39.50 & 15.18 & 15.30 & 16.02 & 22.80 & 14.45 \\
\hline & Cheyletidae & $\begin{array}{l}\text { Cheyletus malaccensis } \\
\text { (Oudmans) }\end{array}$ & 19.80 & 14.43 & 35.70 & 13.72 & 15.10 & 15.81 & 24.00 & 15.21 \\
\hline & & Cheyletus eruditus(Oudmans) & 4.80 & 3.49 & 15.10 & 5.80 & 2.30 & 2.41 & 8.50 & 5.38 \\
\hline & Phytoseiidae & Amblyseius sp. & 21.50 & 15.67 & 41.70 & 16.03 & 15.40 & 16.12 & 23.90 & 15.14 \\
\hline & Macrochelidae & Macrocheles sp. & 20.60 & 15.01 & 33.80 & 12.99 & 14.50 & 15.18 & 21.10 & 13.37 \\
\hline & Rhadacaridae & Rhodacarus sp. & 19.00 & 13.85 & 30.80 & 11.84 & 13.30 & 13.93 & 20.90 & 13.24 \\
\hline & Ascidae & Blattiosocius sp. & 11.90 & 8.67 & 25.30 & 9.72 & 8.30 & 8.69 & 15.10 & 9.56 \\
\hline \multirow{14}{*}{ 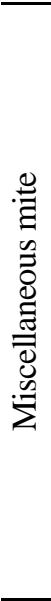 } & Haplozetidae & Haplozetes & 26.50 & 6.05 & 51.80 & 7.08 & 18.60 & 6.55 & 31.40 & 6.88 \\
\hline & Hypochthoniidae & Hypochthnt & 19.30 & 4.41 & 37.20 & 5.08 & 13.60 & 4.79 & 22.60 & 4.95 \\
\hline & Domacidae & Belba sp. & 18.90 & 4.32 & 32.00 & 4.37 & 9.40 & 3.31 & 20.10 & 4.41 \\
\hline & Galumnidae & Galumna sp. & 1.30 & 0.29 & 6.80 & 0.93 & 0.80 & 0.28 & 1.60 & 0.35 \\
\hline & Lohmannidae & Lohmannia sp. & 35.20 & 8.04 & 55.10 & 7.53 & 22.40 & 7.89 & 34.10 & 7.47 \\
\hline & Oribatulidae & Oribatula sp. & 30.60 & 6.98 & 51.20 & 6.99 & 20.70 & 7.29 & 33.80 & 7.41 \\
\hline & & Scheloribates sp. & 33.90 & 7.74 & 60.60 & 8.28 & 22.80 & 8.03 & 36.70 & 8.05 \\
\hline & Phthi & Phthiracar & 34.10 & 7.78 & 64.00 & 8.75 & 22.20 & 7.82 & 35.60 & 7.80 \\
\hline & Epilohmanidae & Epilohmania sp. & 33.30 & 7.60 & 56.80 & 7.76 & 23.40 & 8.24 & 34.40 & 7.54 \\
\hline & Tarsonemidae & Tarsonemus sp. & 38.70 & 8.84 & 60.80 & 8.31 & 26.00 & 9.16 & 41.00 & 8.98 \\
\hline & Tydeidae & Tydeus sp. & 45.40 & 10.36 & 69.30 & 9.47 & 28.40 & 10.00 & 45.70 & 10.02 \\
\hline & Pygmephoridae & Pygmephorus sp. & 49.50 & 11.30 & 72.90 & 9.96 & 28.80 & 10.14 & 47.20 & 10.35 \\
\hline & Ameroseiidae & Kleemannia plumosus(Oud.) & 35.10 & 8.01 & 55.20 & 7.55 & 22.10 & 7.78 & 36.30 & 7.96 \\
\hline & Tyroglyphidae & Tyrophagus putrescentiae (Sch.) & 36.10 & 8.24 & 57.80 & 7.90 & 24.70 & 8.70 & 35.60 & 7.80 \\
\hline \multirow{5}{*}{$\begin{array}{l}\frac{\pi}{0} \\
\frac{8}{0} \\
\frac{0}{0} \\
ن\end{array}$} & Onychiuridae & Onychurius sp. & 65.10 & 19.15 & 85.70 & 18.70 & 39.10 & 20.01 & 67.70 & 20.45 \\
\hline & Sphaeridae & Tulbergia sp. & 65.00 & 19.12 & 91.00 & 19.86 & 39.20 & 20.06 & 64.80 & 19.57 \\
\hline & Hypogastruridae & Hypogastrura sp. & 66.20 & 19.49 & 91.40 & 19.95 & 35.70 & 18.27 & 63.30 & 19.12 \\
\hline & Poduridae & Proistoma sp. & 78.30 & 23.04 & 104.60 & 22.83 & 42.30 & 21.65 & 71.90 & 21.71 \\
\hline & Entombyridae & Entombyra sp. & 65.30 & 19.21 & 85.50 & 18.65 & 39.10 & 20.01 & 63.40 & 19.15 \\
\hline
\end{tabular}

3. Population density of mites and Collembola on four ratios of Nodulation and $\mathrm{N}$ fertilization, on cowpea field in soil:

Data in Table (2), showed that the population of predacious mite, miscellaneous mites and Collembola started to appear with high numbers in Nodulation+ $100 \% \mathrm{~N}$ - fertilization, as follow ; $37.33 \%, 37.16 \%$ and $32.95 \%$ during season 2010.While ,during season 2011 those were $39.98 \%, 38.31 \%$ and $34.59 \%$ respectively .On the other hand, in Non- Nodulation+ $50 \%$ Nfertilization appeared with lowest number as follow; $17.23 \%, 16.07 \%$ and $15.21 \%$ during season 2010. While , during season 2011 were $14.67 \%, 14.86 \%$ and 14.75 respectively. It was observed that the miscellaneous mites were higher than Collembola and predacious mite in all nodulation and $\mathrm{n}$ - fertilization, during seasons $2010 \& 2011$. Generally ,it is worth to mention that the highest number of soil mites was recorded in case of nodulation plants, and in addition to the highest rate of nitrogen, similar results were recorded by Abo-Korah et al., (1984-1985a,b and 1985). .Saleh and Tadros (1985). Sharshir (1986), Rosche (1992) and Gamieh and Saadon (1995 a and b) in different crops. They recorded a positive relationship between count of soil mites and gradual increase of nitrogenous fertilization.

4. Population density of soil mites and Collembola under cowpea plants as affected by Nodulation and N- fertilization during (June - July August) months (2010\&2011).

As shown in Table (3), it was found that the population of predacious mite, miscellaneous mites and Collembola started to appear with high number in Nodulation+ $100 \% \mathrm{~N}$ - fertilization during June , July and August(2010) as follow ; $(45.51 \%, 38.01 \%$ and $41.15 \%),(35.56 \%, 33.71 \%$ and $30.36 \%)$; $(38.95 \%, 46.39 \%$ and $36.19 \%)$, respectively . While, during season 2011, those were ( $43.98 \%, 41.34 \%$ and $43.43 \%)$; $(38.31 \%, 35.17 \%$ and $31.82 \%)$; $(41.54 \%, 43.73 \%$ and $33.68 \%)$ respectively . On the other hand, in Non- Nodulation+ $50 \% \mathrm{~N}$ fertilization appeared with lowest number as follow; $(9.63 \%, 17.03 \%$ and $12.34 \%) ;(18.00 \%, 18.37 \%$ and $16.13 \%) ;(18.23 \%, 9.38 \%$ and 14.07$)$ during season 2010.While, during season 2011 those were 
$(11.62 \%, 14.21 \%$ and $11.02 \%) ;(15.45 \%, 16.91 \%$ and $15.83 \%) ;(14.71 \%, 10.56 \%$ and $14.02 \%)$. It was obvious that the miscellaneous mite were high than
Collembola and predacious mite in all Nodulation and N- fertilization, during both season 2010 \& 2011.

Table 2. Population density of soil mites and collmebola under Cowpea plants as affected by Nodulation and Nfertilization.

\begin{tabular}{|c|c|c|c|c|c|c|c|}
\hline \multirow[b]{2}{*}{ Treatmen } & & \multicolumn{3}{|c|}{ Season2010 } & \multicolumn{3}{|c|}{ Season 2011} \\
\hline & & $\begin{array}{c}\text { predacious } \\
\text { mite }\end{array}$ & $\begin{array}{l}\text { Miscellaneous } \\
\text { mites }\end{array}$ & Collembola & $\begin{array}{c}\text { predacious } \\
\text { mite }\end{array}$ & $\begin{array}{c}\text { miscellaneous } \\
\text { mites }\end{array}$ & Collembola \\
\hline Nodulated+ & Mean & 73.90 & 308.20 & 285.70 & 137.20 & 437.90 & 339.90 \\
\hline $50 \% \mathrm{~N}-$ & $\%$ & 11.06 & 46.15 & 42.78 & 14.99 & 47.86 & 37.15 \\
\hline $\begin{array}{l}\text { fertilization } \\
\text { (40kg/feddan) }\end{array}$ & $\%$ & 24.03 & 24.46 & 27.43 & 21.08 & 22.93 & 25.66 \\
\hline Nodulated+ & Mean & 114.80 & 468.30 & 343.30 & 260.20 & 731.50 & 458.20 \\
\hline $100 \% \mathrm{~N}-$ & $\%$ & 12.39 & 50.55 & 37.06 & 17.95 & 50.45 & 31.60 \\
\hline $\begin{array}{l}\text { fertilization } \\
\text { (80kg/feddan) }\end{array}$ & $\%$ & 37.33 & 37.16 & 32.95 & 39.98 & 38.31 & 34.59 \\
\hline Non- & Mean & 53.00 & 202.60 & 158.50 & 95.50 & $283 . .90$ & 195.40 \\
\hline Nodulated+ & $\%$ & 12.79 & 48.92 & 38.27 & 16.61 & 49.39 & 33.99 \\
\hline $\begin{array}{l}50 \% \mathrm{~N}- \\
\text { fertilization } \\
\text { (40kg/feddan) }\end{array}$ & $\%$ & 17.23 & 16.07 & 15.21 & 14.67 & 14.86 & 14.75 \\
\hline Non- & Mean & 65.80 & 280.90 & 254.20 & 157.80 & 456.10 & 331.10 \\
\hline Nodulated+ & $\%$ & 10.95 & 46.75 & 42.30 & 16.69 & 48.26 & 35.04 \\
\hline $\begin{array}{l}100 \% \mathrm{~N}- \\
\text { fertilization } \\
\text { (80kg/feddan) }\end{array}$ & $\%$ & 21.39 & 22.29 & 24.40 & 24.25 & 23.88 & 24.99 \\
\hline
\end{tabular}

Table 3. Population density of soil mites and collmebola under Cowpea plants during June, July and August as affected by Nodulation and $\mathrm{N}$ - fertilization.

\begin{tabular}{|c|c|c|c|c|c|c|c|c|}
\hline \multirow{14}{*}{$\stackrel{\Xi}{\Xi}$} & \multirow[b]{2}{*}{ Treatment } & & \multicolumn{3}{|c|}{ Season 2010} & \multicolumn{3}{|c|}{ Season 2011} \\
\hline & & & $\begin{array}{l}\text { predacious } \\
\text { mite }\end{array}$ & $\begin{array}{c}\text { Miscellaneo } \\
\text { us mites }\end{array}$ & Collembola & $\begin{array}{c}\text { predacious } \\
\text { mite }\end{array}$ & $\begin{array}{c}\text { miscellaneous } \\
\text { mites }\end{array}$ & Collmebola \\
\hline & \multirow{3}{*}{$\begin{array}{l}\text { Nodulated+ } 50 \% \mathrm{~N}- \\
\text { fertilization } \\
(40 \mathrm{~kg} / \text { feddan })\end{array}$} & Mean & 16.00 & 59.25 & 32.75 & 41.25 & 129.75 & 66.25 \\
\hline & & $\%$ & 14.81 & 54.86 & 30.32 & 17.38 & 54.68 & 27.92 \\
\hline & & $\%$ & 21.26 & 20.81 & 26.95 & 16.53 & 17.89 & 21.47 \\
\hline & \multirow{3}{*}{$\begin{array}{l}\text { Nodulated+ } \\
100 \% \mathrm{~N}- \\
\text { fertilization } \\
(80 \mathrm{~kg} / \text { feddan })\end{array}$} & Mean & 34.25 & 108.25 & 50.00 & 109.75 & 299.75 & 134.00 \\
\hline & & $\%$ & 17.79 & 56.23 & 25.97 & 20.19 & 55.15 & 24.65 \\
\hline & & $\%$ & 45.51 & 38.01 & 41.15 & 43.98 & 41.34 & 43.43 \\
\hline & \multirow{3}{*}{$\begin{array}{l}\text { NonNodulated }+5 \\
\text { N- fertilization } \\
(40 \mathrm{~kg} / \text { feddan }) \\
\end{array}$} & Mean & 7.25 & 48.50 & 15.00 & 29.00 & 103.00 & 34.00 \\
\hline & & $\%$ & 10.25 & 68.55 & 21.20 & 17.46 & 62.05 & 20.48 \\
\hline & & $\%$ & 9.63 & 17.03 & 12.34 & 11.62 & 14.21 & 11.02 \\
\hline & \multirow{3}{*}{$\begin{array}{l}\text { NonNodulated }+100 \\
\% \mathrm{~N}-\text { fertilization } \\
(80 \mathrm{~kg} / \text { feddan })\end{array}$} & Mean & 17.75 & 68.75 & 23.75 & 69.50 & 192.50 & 74.25 \\
\hline & & $\%$ & 16.09 & 62.36 & 21.54 & 20.66 & 57.25 & 22.08 \\
\hline & & $\%$ & 23.58 & 24.14 & 19.55 & 27.85 & 26.55 & 24.06 \\
\hline \multirow{12}{*}{$\stackrel{\lambda}{\Xi}$} & \multirow{3}{*}{$\begin{array}{l}\text { Nodulated+ } 50 \% \mathrm{~N}- \\
\text { fertilization } \\
(40 \mathrm{~kg} / \text { feddan })\end{array}$} & Mean & 127.75 & 524.00 & 428.50 & 219.50 & 669.25 & 486.50 \\
\hline & & $\%$ & 11.82 & 48.51 & 39.66 & 15.96 & 48.66 & 35.37 \\
\hline & & $\%$ & 24.93 & 24.91 & 27.64 & 22.60 & 23.71 & 26.26 \\
\hline & \multirow{3}{*}{$\begin{array}{l}\text { Nodulated+ } \\
100 \% \mathrm{~N}- \\
\text { fertilization } \\
(80 \mathrm{~kg} / \text { feddan })\end{array}$} & Mean & 182.25 & 709.00 & 470.75 & 372.00 & 992.50 & 589.50 \\
\hline & & $\%$ & 13.38 & 52.05 & 34.56 & 19.04 & 50.79 & 30.16 \\
\hline & & $\%$ & 35.56 & 33.71 & 30.36 & 38.31 & 35.17 & 31.82 \\
\hline & \multirow{3}{*}{$\begin{array}{l}\text { NonNodulated+ } \\
50 \% \mathrm{~N} \text { - fertilization } \\
(40 \mathrm{~kg} / \text { feddan })\end{array}$} & Mean & 92.25 & 386.50 & 250.00 & 150.00 & 477.25 & 293.25 \\
\hline & & $\%$ & 12.66 & 53.04 & 34.30 & 16.29 & 51.85 & 31.86 \\
\hline & & $\%$ & 18.00 & 18.37 & 16.13 & 15.54 & 16.91 & 15.83 \\
\hline & \multirow{3}{*}{$\begin{array}{l}\text { NonNodulated+ } \\
100 \% \mathrm{~N}- \\
\text { fertilization } \\
\text { ( } 80 \mathrm{~kg} / \text { feddan) }\end{array}$} & Mean & 110.25 & 483.75 & 401.00 & 229.50 & 682.75 & 483.50 \\
\hline & & $\%$ & 11.08 & 48.62 & 40.30 & 16.44 & 48.92 & 34.64 \\
\hline & & $\%$ & 21.51 & 23.00 & 25.86 & 23.63 & 24.19 & 26.09 \\
\hline
\end{tabular}


Table 3. Continue

\begin{tabular}{|c|c|c|c|c|c|c|c|c|}
\hline & & & & Season201 & & & Season 20 & \\
\hline & Treatment & & $\begin{array}{c}\text { Predac } \\
\text { ious } \\
\text { mite }\end{array}$ & $\begin{array}{l}\text { Miscellaneous } \\
\text { mites }\end{array}$ & collembola & $\begin{array}{c}\text { predacious } \\
\text { mite }\end{array}$ & $\begin{array}{l}\text { miscellaneous } \\
\text { mites }\end{array}$ & Collembola \\
\hline & Nodulated $+50 \% \mathrm{~N}-$ & Mean & 41.00 & 187.25 & 253.00 & 52.25 & 295.75 & 297.00 \\
\hline & fertilization (40 kg/feddan) & $\%$ & 8.52 & 38.91 & 52.57 & 12.18 & 43.81 & 44.00 \\
\hline & & $\%$ & 22.65 & 24.57 & 27.13 & 20.25 & 24.11 & 25.82 \\
\hline$\tilde{b}_{0}^{\infty}$ & Nodulated+100\%N- & Mean & 70.50 & 353.50 & 337.50 & 168.75 & 536.50 & 422.00 \\
\hline$\stackrel{\vec{Z}}{4}$ & fertilization ( $80 \mathrm{~kg} /$ feddan) & $\%$ & 9.26 & 46.42 & 44.32 & 14.97 & 47.59 & 37.44 \\
\hline & & $\%$ & 38.95 & 46.39 & 36.19 & 41.54 & 43.73 & 33.68 \\
\hline & NonNodulated $+50 \% \mathrm{~N}-$ & Mean & 33.00 & 71.50 & 131.25 & 59.75 & 129.50 & 161.25 \\
\hline & fertilization ( $40 \mathrm{~kg} /$ feddan) & $\%$ & 13.99 & 30.33 & 55.67 & 17.05 & 36.94 & 46.00 \\
\hline & & $\%$ & 18.23 & 9.38 & 14.07 & 14.71 & 10.56 & 14.02 \\
\hline & NonNodulated $+100 \%$ Nfertili & Mean & 36.50 & 149.75 & 210.75 & 95.50 & 265.00 & 270.00 \\
\hline & zation (80kg/feddan) & $\%$ & 9.19 & 37.72 & 53.08 & 15.15 & 42.03 & 42.83 \\
\hline & & $\%$ & 20.16 & 19.65 & 22.60 & 23.51 & 21.60 & 23.47 \\
\hline
\end{tabular}

\section{Shannon-Weaner diversity index (S.W.I) of predacious mite, miscellaneous mite and Collembola:}

\section{a. In the first season 2010 :}

Data in Table (4) presented the computed values of the Shannon-weaner diversity index in relation to cowpea in which samples were taken. The S.W.I for predatory mite in the sampled cowpea was highest in Non- Nodulated+ $50 \% \mathrm{~N}$ - fertilization, Nodulated $+50 \% \quad \mathrm{~N}$ - fertilization and NonNodulated $+100 \% \mathrm{~N}$ - fertilization being 0.9330 , 0.8638 and 0.8525 . respectively. while in Nodulated $+50 \% \mathrm{~N}$ - fertilization the lowest value was 0.8456 .The number of species were 8 species for the Nodulation \& Non - Nodulation+ Nfertilization. the S.W diversity index for miscellaneous mites in the sampled cowpea was highest in Non- Nodulated+ $50 \% \mathrm{~N}$ - fertilization, Nodulated $+50 \% \mathrm{~N}$ - fertilization and Nodulated+ $50 \% \mathrm{~N}$ - fertilization being $2.7336,2.1411$ and 1.0919 respectively, while, in Non -Nodulated+ $100 \% \mathrm{~N}$ - fertilization, it was the lowest, being 1.0888 , The number of species were 14 and 13 species for the Nodulation \& Non - Nodulation+ Nfertilization .Also the S.W. diversity index for springtails in the sampled cowpea was highest in Non- Nodulation+ $100 \% \quad \mathrm{~N}$ - fertilization, NonNodulation+ $50 \% \mathrm{~N}$ - fertilization and Nodulated+ $50 \% \mathrm{~N}$ - fertilization, being $0.6985,0.6978$ and 0.6974 respectively ; While, in Nodulated $+100 \% \mathrm{~N}$ fertilization it was the lowest, being 0.6966 . The number of species were 5 species for the former Nodulation+ $\mathrm{N}$ - fertilization . It could be concluded that cowpea has dominant and subdominant predacious mite, miscellaneous mite and springtails species which can affect the diversity index values .The relationship between species and ecological process can change quantitatively and quantitatively with environment context (Cardinale \& Nelson ,1999).

\section{B. in the second season 2011:}

Data in Table (4) revealed That S.W. diversity index for predatory mite species was the highest in Nodulated $+100 \% \mathrm{~N}$ - fertilization, Non-Nodulated+ $100 \% \mathrm{~N}$ - fertilization and Nodulated+ $50 \% \mathrm{~N}$ fertilization being $0.8874,0.8866$ and 0.8753 respectively . While, in Non- Nodulated+ $50 \% \mathrm{~N}-$ fertilization, it was the lowest, being 0.8659.The number of species were 8 species for the former Nodulation and $\mathrm{N}$ - fertilization. Also, the S.W. diversity index for miscellaneous mite species was the highest in Nodulated $+100 \% \mathrm{~N}$ - fertilization, Non- Nodulated $+100 \% \mathrm{~N}$ - fertilization and NonNodulated $+50 \% \mathrm{~N}$ - fertilization being 1.1169 , 1.1096 and 1.1048 respectively, while ,in Nodulated $+50 \% \mathrm{~N}$ - fertilization, it was the lowest, being 1.1047. The number of species was 14 species for the former Nodulation and $\mathrm{N}$ - fertilization .On the other hand, the S.W. diversity index for springtails in the sampled cowpea was highest in Non- Nodulation+ $100 \% \mathrm{~N}$ - fertilization, NonNodulated $+50 \% \mathrm{~N}$ - fertilization and Nodulated+ $50 \% \mathrm{~N}$ - fertilization being $0.6984,0.6981$ and 0.6977 respectively, while, in Nodulated $+100 \% \mathrm{~N}-$ fertilization it was the lowest 0.6976 . The number of species were 5 species for the former Nodulation and N-fertilization. Similar results were obtained by El - Dakhakhni et al.(1995)who reported that clover had the highest number of beneficial insect species, was 22 while, cotton and soybean had the lowest number , 12 for each one .The S.W. for natural enemy species in the sampled crops was the highest in clover being 2.52 while in maize it was the lowest being 1.81.As for cotton and soybean, it was 2.08 and 2.41 respectively. Also, El-Mezayyen (2001) indicated that S.W. diversity index for pests , beneficial and visitors species in the sampled crops was the highest in alfalfa at sebha being 1.664, 0.6129 and 0.8222 while in the Egyptian clover at Kafr El- sheikh, it was the lowest, being 0.6154, 0.5152 and 1.1480 .The number of species were 14,11 and 5 for alfalfa. While, they were 13,8 and 6 
for the Egyptian clover respectively, in the current study, it was anticipated that the relative predacious mite, miscellaneous mite and springtails abundance of species on cowpea crop may have an effect on diversity index values of S.W. support such suggestion.

Table 4. Shannon-weaner index as computed from related species of predacious mite, miscellaneous mite and Collembola from Cowpea field as affected by Nodulation and N-fertilization during seasons 2010\&2011.

\begin{tabular}{|c|c|c|c|c|c|c|c|c|c|}
\hline \multirow[t]{3}{*}{ Treatment } & \multicolumn{3}{|c|}{ Predacious mite } & \multicolumn{3}{|c|}{ Miscellaneous mite } & \multicolumn{3}{|c|}{ Collembola } \\
\hline & \multicolumn{6}{|c|}{ (2010) } & \multirow[b]{2}{*}{$\begin{array}{c}\text { Mean } \\
\text { no }\end{array}$} & \multirow[b]{2}{*}{$\begin{array}{c}\text { No. of } \\
\text { species }\end{array}$} & \multirow[b]{2}{*}{ SW } \\
\hline & $\begin{array}{c}\text { Mean } \\
\text { no }\end{array}$ & $\begin{array}{l}\text { No. of } \\
\text { species }\end{array}$ & SW & $\begin{array}{c}\text { Mean } \\
\text { no }\end{array}$ & $\begin{array}{l}\text { No. of } \\
\text { species }\end{array}$ & SW & & & \\
\hline $\begin{array}{l}\text { Nodulated }+50 \% \mathrm{~N}- \\
\text { fertilization } \\
(40 \mathrm{~kg} / \text { feddan })\end{array}$ & 73.90 & 8 & 0.8456 & 308.20 & 14 & 2.1411 & 285.70 & 5 & 0.6974 \\
\hline $\begin{array}{l}\text { Nodulated }+100 \% \mathrm{~N}- \\
\text { fertilization } \\
(80 \mathrm{~kg} / \text { feddan }) \\
\end{array}$ & 114.80 & 8 & 0.8638 & 468.30 & 14 & 1.0919 & 343.30 & 5 & 0.6966 \\
\hline $\begin{array}{l}\text { NonNodulated }+50 \% \mathrm{~N}- \\
\text { fertilization } \\
(40 \mathrm{~kg} / \text { feddan })\end{array}$ & 53.00 & 8 & 0.9330 & 202.60 & 14 & 2.7336 & 158.50 & 5 & 69780. \\
\hline $\begin{array}{l}\text { NonNodulated }+100 \% \mathrm{~N}- \\
\text { fertilization } \\
(80 \mathrm{~kg} / \text { feddan })\end{array}$ & 65.80 & 8 & 0.8525 & 280.90 & 14 & 1.0888 & 254.20 & 5 & 0.6985 \\
\hline \multicolumn{10}{|c|}{2011} \\
\hline $\begin{array}{l}\text { Nodulated }+50 \% \mathrm{~N}- \\
\text { fertilization } \\
(40 \mathrm{~kg} / \text { feddan })\end{array}$ & 137.20 & 8 & 0.8753 & 437.90 & 14 & 1.1047 & 339.90 & 5 & 0.6977 \\
\hline $\begin{array}{l}\text { Nodulated }+100 \% \mathrm{~N}- \\
\text { fertilization } \\
(80 \mathrm{~kg} / \text { feddan })\end{array}$ & 260.20 & 8 & 0.8874 & 731.50 & 14 & 1.1169 & 458.20 & 5 & 0.6976 \\
\hline $\begin{array}{l}\text { NonNodulated }+50 \% \mathrm{~N}- \\
\text { fertilization } \\
(40 \mathrm{~kg} / \text { feddan })\end{array}$ & 95.50 & 8 & 0.8659 & 283.90 & 14 & 1.1048 & 195.40 & 5 & 0.6981 \\
\hline $\begin{array}{l}\text { NonNodulated }+100 \% \mathrm{~N}- \\
\text { fertilization } \\
(80 \mathrm{~kg} / \text { feddan })\end{array}$ & 157.80 & 8 & 0.8866 & 456.10 & 14 & 1.1096 & 331.10 & 5 & 0.6984 \\
\hline
\end{tabular}

\section{References}

Abdel-Alim , A. A (1994). Ecology studied on soil Acari in Cowpea field in Minia . J. Agric. Res.\&Dev . 2(10): 261-273 pp.

Abo-Korah, S. M.; A.A.Osman and S.E. Saadon (1984-1985a). Effect of certain soil fertilization on soil Acari inhabiting okra field in Menufiya Governorate ,Egypt .Bull .Soc. ent. Egypte . 65: 59-64.

Abo-Korah, S. M.; A.A.Osman and S.E. Saadon (1984-1985b). Influence of certain soil fertilizer in okra field on Cohor Tarsoneminal (Acari:Heterostigmata)in Menufiya Governorate ,Egypt .Bull .Soc. ent. Egypte . 65: 139-143 pp.

Abo-Korah, S. M.; H.S.A.Radwan ;M.R. Abo ElGhar and S.E. Saadon (1985). Response of Acari groups cartain fertilizer adopted in cotton fields in Menufiya Governorate,Egypt .proc . Egypt's National Con. Ent.Dec.6-9 ,1982.1:125131.
Abo-Tayesh, M.A.M. (2008). Survey of soil Acarina and Collembola inhabiting onion (Allium cepa, L.) at two locations in Egypt. J. Agric. Res., Kafr El-Sheikh Univ., 34(4): 1020-1034.

Abo-Tayesh, M.A.M. and E.A.Moursi (2013). Population miscellaneous mite and Collembolan associated with manures in tomato soil at Menufiya Governorate.Egypt. J .Plant.Pro.Res.(volume 1No(4)) October 2013.15-30 PP.

Abo-Tayesh, M.A.M.; M.A.Khatab;A.B.Abo.ElKassem and E.H.El-Kasser (2013).Effect of some organic manures application on number of predacious mite in soil of tomato Population miscellaneous mite and Collembolan associated with manures in tomato crop in Menufiya Governorate,Egypt. J.Plant.Pro.Res.(volume 1No(4)) October 2013.31-44 PP.

Boraei, H.A.;M. S.Tadros;M.B.Shawer and G.N.Gamieh (1994). Survey on mite associated with Orchared treas at Kafer El- Sheikh. Governorate.Bull .zool .Soc.Egypt. (42)).55-68 . 
Cardinale, B.J. and K. Nelson (1999). The effects of spatial variation and disturbance on the relationship between species diversity and ecosystem function. Ecol. Soc. Amer. 84 ${ }^{\text {th }}$ Ann. Meet. 12, Abstract.

El-Dakhakhni, T.N.; I.S. El-Hawary; M.S. Tadros; M.B. Shawer and G.A. El-Mezayyen (1995). Studies on the beneficial insects on the Egyptian clover, cotton, maize and soybean at Kafr El-Sheikh. Agric. Sci., Mansoura Univ., 20(6): 3125-3133.

El-Hawary, T.S.; T.N. El-Dakhakhni; M.B. Shawer; M.S. Tadros and G.A. El-Mezayyen (1995). Insect pests of the Egyptian clover, cotton, maize and soybean at Kafr El-Sheikh distinct Egypt .J. Agric. Sci., Mansoura Univ., 20(6): 3097-3105.

El-Kifl, A.H. (1957). The soil arthropod fauna in a farm of Giza, U.A.R. Bull Soc. Ent. Egypte, 41: 231-268.

El-Kifl, A.H. (1968). The soil arachnoidae of a farm at Giza, U.A.R. Bull. Soc. ent. Egypte, 52: 413428.

El-Mezayyen, G.A. (2001). Field studies on the insect species on two forage crops, the Egyptian clover in Egypt and alfalfa in Libya. Egypt. J. Agric., 79(3): 983-934.

Gamieh, G.N. and S.E.Saadon (1995)a . Population density of mite in saybean Fields as affected by Nodulation, coating seeds with trace elements and nitrogenous fertilization . J. Agric.Res . Tanta univ .21(1): 165-173.

Gamieh, G.N. and S.E.Saadon (1995)b . The effect of Nodulation, $\mathrm{N}$ - fertilization and certain acaricide on phytophagous and soil mite in soybean fields . J. Agric.Res, Tanta univ .24(2): 137-149.

Hassan , M.M.;M.M.Tantawy;F.K.Aly and G.A.I.Karaman (1985). Contributions of the effect of macro elements and insecticides on soil mites and sucking insects and yield components of cowpea .Minia. J. Agric.Res. \&Dec .7: 382389.

Kerbs, C.J. (1978). Ecology "The experimental analysis distribution and abundance, $2^{\text {nd }}$ ed. (Harper \& Row Pub.), NY, USA, 678 pp.

Price, P.W. (1984). Insect Ecology, $2^{\text {nd }}$ edition (J. Wiley Sons Inc. ed., Wiley Interscience Publication (Publ.), USA, 607 pp.

Rosche , O. (1992). Influence of nitrogen fertilizer on the structure of soil micro arthropods communities in an Agro - Ecosystem .XI-Int. Colloq on soil zoology, soil organisms and soil Health Jyvaskyla, Finland, 10-14 August Abstract, p.191.

Saleh , R.S. and M.S. Tadros (1985). The effect of some foliar fertilizers on both soil arthropods and Gladulis hortul .Prac. IX.Int Collog .Soil Zoo. Moscow.,USSR. Abstract P. 239.

Sarshir , F.A.A. (1986). Studied on some ecological factors affecting the population density of soil Acaring in Kafr El-Sheikh region .M.SC. Thesis .Tanta univ .150pp.

Tadros, M.S. (1975). Ecological studies on soil oribatids in Kafr El-Sheikh region, A.R.E. Bull. Zool. Soc. Egypt., 27: 85-89. 


\title{
تأثير التلقيح البكتيري والتسميد الأزوتى على كل من الأكاروسات و الكولمبولا في الترية في حقول اللوبيا فى محافظة كفر الثيخ
}

\author{
محسن عطية أبو طايش \\ معهز بحوث وقاية النباتات ـ مركز البحوث الزراعية ـ مصر
}

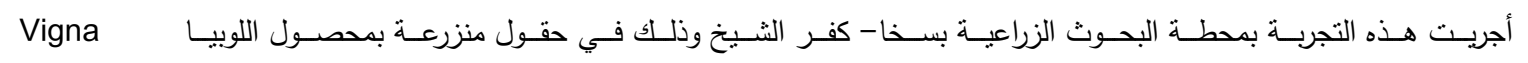
بnguiculata(L)walp.

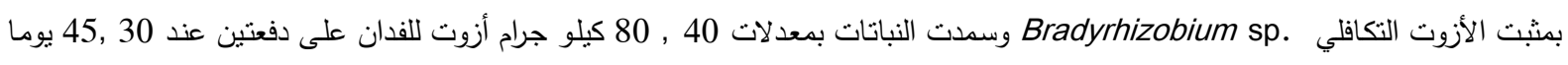

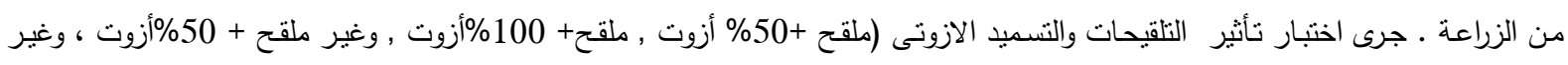

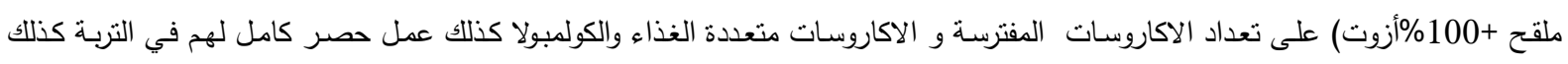

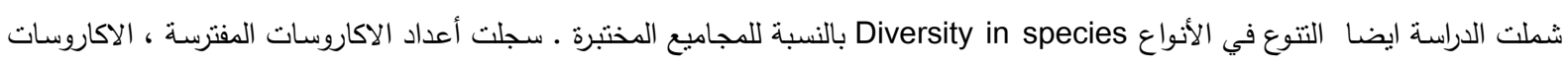

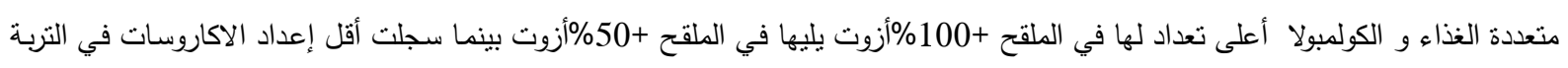

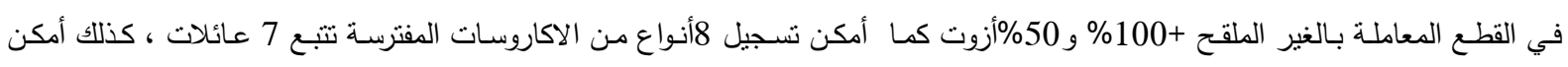

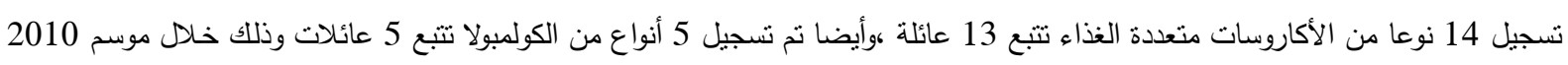

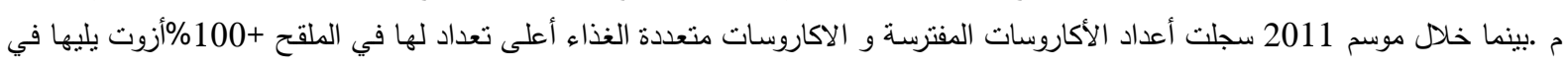

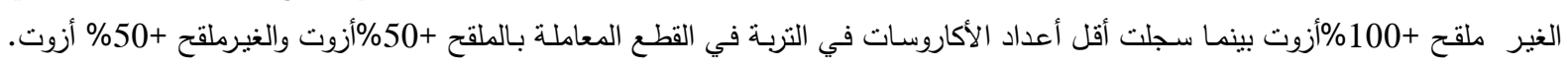

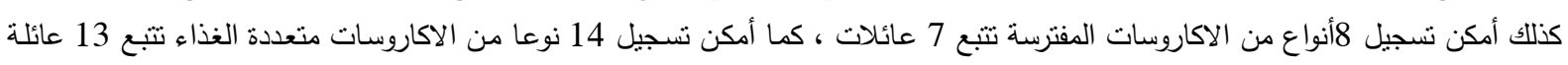

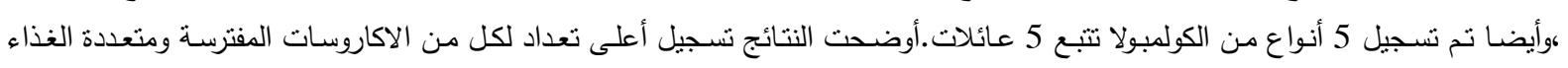

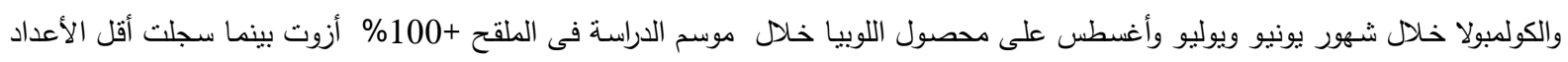

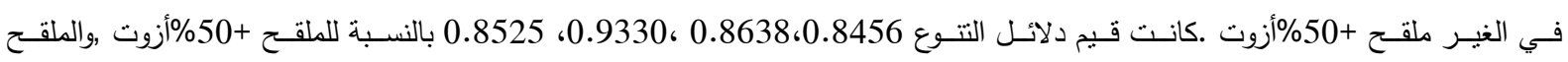

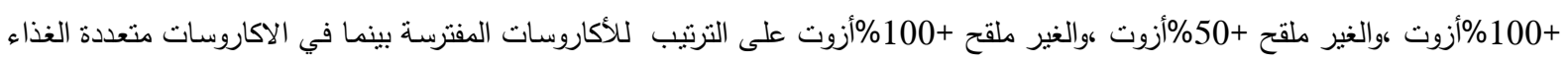

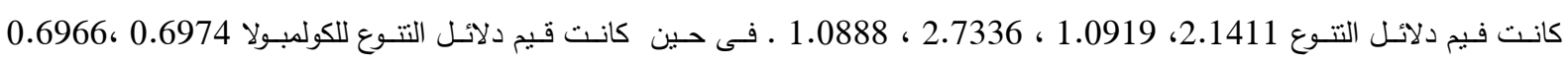

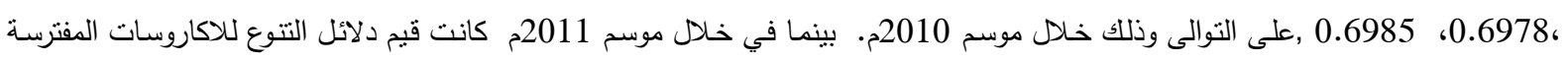

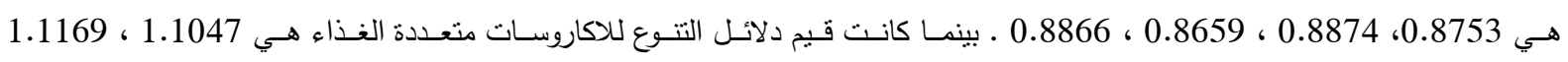

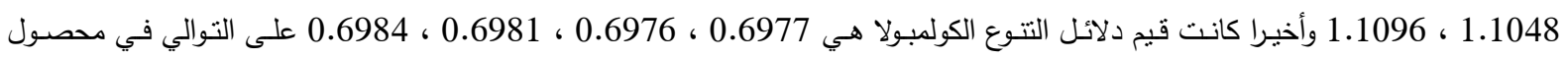
اللوبيا. وجد أيضا أن التواجد النسبي لأنواع الاكاروسات المفترسة و الاكاروسات متعددة الغذاء والكولمبولا في أرض اللوبيا لها تأثير على قليم دليل التنوع • 